This item was submitted to Loughborough's Research Repository by the author.

Items in Figshare are protected by copyright, with all rights reserved, unless otherwise indicated.

\title{
Coordinated standoff tracking of in- and out-of-surveillance targets using constrained particle filter for UAVs
}

PLEASE CITE THE PUBLISHED VERSION

http://dx.doi.org/10.1109/IVS.2015.7225734

PUBLISHER

(C) IEEE

VERSION

AM (Accepted Manuscript)

LICENCE

CC BY-NC-ND 4.0

\section{REPOSITORY RECORD}

Oh, Hyondong, Cunjia Liu, Seungkeun Kim, Hyo-Sang Shin, and Wen-Hua Chen. 2015. "Coordinated Standoff Tracking of In- and Out-of-surveillance Targets Using Constrained Particle Filter for Uavs". figshare. https://hdl.handle.net/2134/18439. 


\title{
Coordinated Standoff Tracking of In- and Out-of-Surveillance Targets Using Constrained Particle Filter for UAVs
}

\author{
Hyondong Oh, Cunjia Liu, Seungkeun Kim, Hyo-Sang Shin, and Wen-Hua Chen
}

\begin{abstract}
This paper presents a new standoff tracking framework of a moving ground target using UAVs with a limited sensing capability such as sensor field-of-view and motion constraints. To maintain persistent track of the target even in case of target loss (out of surveillance) for a certain period, this study predicts the target existence area using the particle filter, and produces control commands to ensure that all predicted particles can be covered by the field-of-view of the UAV sensor at all times. To improve target prediction/estimation accuracy, the road information is incorporated into the constrained particle filter where the road boundaries are modelled as nonlinear inequality constraints. Both Lyapunov vector field guidance and nonlinear model predictive control methods are applied for the standoff tracking and phase angle control, and the advantages and disadvantages of them are compared using numerical simulation results.
\end{abstract}

\section{INTRODUCTION}

The operation of a team of UAVs (Unmanned Aerial Vehicle) has proliferated in recent decades in both military and civilian missions. Surveillance and subsequent tracking, of a moving ground target of interest is one of the important capabilities of the UAV required to accomplish those missions successfully while increasing an overall knowledge of target's intent and surrounding environments.

To this end, state estimation of the target of interest is essential by using appropriate tracking filter design with available sensor data and domain knowledge. Since ground vehicles are generally moving on road networks whose topographical coordinates could be obtained, such road-map information can be used to improve the estimation accuracy significantly by constraining the state of the target within the road geometry [1]-[3]. While estimating states of the target, UAVs also should be able to follow the target persistently. This is not an easy task due to unknown movements of the target as well as physical and sensing constraints of the UAV. As one of solutions for this task, a coordinated standoff tracking concept is introduced for UAVs, which is to keep a certain distance (termed standoff distance) from the moving target, resulting in orbiting around the target due to the possible speed superiority of the UAV

This work was supported by the UK Engineering and Physical Science Research Council (EPSRC) under the Grant EP/J011525/1.

Hyondong Oh, Cunjia Liu and Wen-Hua Chen are with the Department of Aeronautical and Automotive Engineering, Loughborough University, Loughborough, Leicestershire, LE11 3TU, United Kingdom $\{\mathrm{h} . \mathrm{oh}$, c.liu5, w.chen\}elboro.ac.uk

Seungkeun Kim is with the Department of Aerospace Engineering, Chungnam National University, 99 Daehak-ro, Yuseong-gu, Daejeon 305764, Republic of Korea skim78@ cnu.ac. kr

Hyo-Sang Shin is with the Institute of Aerospace Sciences, Cranfield University, Cranfield, Bedfordshire, MK43 OAL, United Kingdom h.shin@cranfield.ac.uk over the ground targets. Various approaches for this problem have been proposed: vector fields [4], differential geometry [5], controlled collective motion [6], and nonlinear model predictive control (NMPC) [7]. However, there is relatively little research on the standoff tracking considering the target uncertainty and the limited sensing capability of the UAV.

With above backgrounds, this paper proposes the persistent standoff tracking guidance approach for a moving ground target using the particle filter framework along with roadmap information. Firstly, to estimate the states of the target moving on the road, this paper applies the constrained PF (CPF) by treating the road as an inequality constraint. Then, this paper further extends the previous standoff tracking guidance algorithms: the vector field based guidance [4] and the NMPC approach [7] by considering sensing and physical constraints of UAVs more explicitly. Due to the limited sensing capabilities such as a limited sensor fieldof-view (FOV) or sensing failure from line-of-sight block by obstacles, the target can be in and out of surveillance frequently. Here, by out of surveillance, we mean that the target is lost for certain duration intermittently. To maintain continuous track of the target, prediction of a region within which the moving target is likely to present for a given lead time becomes very important.

To tackle this issue, this study uses the position of particles generated from the CPF to predict possible areas of target existence. By commanding the UAVs to maintain the desired standoff distance determined by target existence area, the UAVs keep all the particles within their sensor FOV, and as a result, the probability of successful target track maintenance can be improved. Although a similar concept has been proposed by Tang and Ozguner [1], their focus was mainly on the target estimation rather than the UAV guidance algorithm. Besides, as the standoff distance is continuously changing according to the predicted target existence area, this study needs to address a variable standoff distance tracking problem, which is different from the previous work using a pre-defined fixed desired standoff distance. Both Lyapunov vector field guidance and NMPC methods are applied for this new standoff tracking problem, and the characteristics of them are compared quantitatively and qualitatively with numerical simulation results.

The structure of this paper is given as follows. Section II presents the constrained particle filter algorithm for ground target localisation and prediction. Section III proposes the application of LVFG and NMPC guidance for the standoff tracking guidance for multiple UAVs with the limited sensing capability using the CPF. Section IV presents numerical sim- 
ulation results of a ground target tracking scenario. Lastly, conclusions and future work are given in Section V.

\section{Constrained Particle Filter}

This section introduces the constrained particle filtering (CPF) algorithm aided by road information and how to utilise the sampled particles from the CPF to predict a possible target existence area.

\section{A. Brief introduction to the constrained PF}

Consider the target dynamics can be described by the following discrete state-space model:

$$
\begin{array}{r}
\mathbf{x}_{k}^{t}=\mathbf{f}\left(\mathbf{x}_{k-1}^{t}\right)+\mathbf{w}_{k} \\
\mathbf{z}_{k}=\mathbf{h}\left(\mathbf{x}_{k}^{t}\right)+\mathbf{v}_{k}
\end{array}
$$

where $\mathbf{x}_{k}^{t}, \mathbf{z}_{k}, \mathbf{w}_{k}$ and $\mathbf{v}_{k}$ are target state, measurement, process noise and measurement noise at time $k$, respectively. The objective of state estimation is to recursively infer the posteriori distribution $p\left(\mathbf{x}_{k}^{t} \mid \mathbf{z}_{1: k}\right)$ given the observation sequence $\mathbf{z}_{1: k}$ and the initial state $\mathbf{x}_{0}^{t}$. Bayesian inference can be used to construct the posteriori. Given the posteriori density $p\left(\mathbf{x}_{k-1}^{t} \mid \mathbf{z}_{1: k-1}\right)$ at time $k-1$, the prior density at time $k$ can be obtained by the prediction step, such that

$$
p\left(\mathbf{x}_{k}^{t} \mid \mathbf{z}_{1: k-1}\right)=\int p\left(\mathbf{x}_{k}^{t} \mid \mathbf{x}_{k-1}^{t}\right) p\left(\mathbf{x}_{k-1}^{t} \mid \mathbf{z}_{1: k-1}\right) \mathrm{d} \mathbf{x}_{k-1}^{t}
$$

Next, an update step can be performed by using the new observation $\mathbf{z}_{k}$ as:

$$
p\left(\mathbf{x}_{k}^{t} \mid \mathbf{z}_{1: k}\right)=\frac{p\left(\mathbf{z}_{k} \mid \mathbf{x}_{k}^{t}\right) p\left(\mathbf{x}_{k}^{t} \mid \mathbf{z}_{1: k-1}\right)}{p\left(\mathbf{z}_{k} \mid \mathbf{z}_{1: k-1}\right)}
$$

Note that this marginalised posteriori density $p\left(\mathbf{x}_{k}^{t} \mid \mathbf{z}_{1: k}\right)$ can also be derived by integrating the following recursion:

$$
p\left(\mathbf{x}_{1: k}^{t} \mid \mathbf{z}_{1: k}\right)=p\left(\mathbf{x}_{1: k-1}^{t} \mid \mathbf{z}_{1: k-1}\right) \frac{p\left(\mathbf{z}_{k} \mid \mathbf{x}_{k}^{t}\right) p\left(\mathbf{x}_{k}^{t} \mid \mathbf{x}_{k-1}^{t}\right)}{p\left(\mathbf{z}_{k} \mid \mathbf{z}_{1: k-1}\right)}
$$

As there is no tractable solution for the above Bayesian estimation when the system is nonlinear/non-Gaussian, a numerical solution, namely the Monte Carlo method, is adopted in particle filters [8]. A target distribution can be approximated by a set of samples and their associated weights drawn from a proposal density. In terms of a posteriori density $p\left(\mathbf{x}_{1: k}^{t} \mid \mathbf{z}_{1: k}\right)$, it can be represented as:

$$
p\left(\mathbf{x}_{1: k}^{t} \mid \mathbf{z}_{1: k}\right)=\sum_{i=1}^{N_{p}} w_{k}^{i} \delta\left(\mathbf{x}_{1: k}^{t}-\mathbf{x}_{1: k}^{t, i}\right)
$$

where $\left\{\mathbf{x}_{1: k}^{t, i}\right\}_{i=1}^{N_{p}}$ is the set of particles of size $N_{p}$ drawn from a proposal density $\pi\left(\mathbf{x}_{1: k}^{t} \mid \mathbf{z}_{1: k}\right),\left\{w_{k}^{i}\right\}_{i=1}^{N_{p}}$ is the corresponding importance weights defined as:

$$
\bar{w}_{k}^{i}=\frac{p\left(\mathbf{x}_{1: k}^{t} \mid \mathbf{z}_{1: k}\right)}{\pi\left(\mathbf{x}_{1: k}^{t} \mid \mathbf{z}_{1: k}\right)}, \quad w_{k}^{i}=\frac{\bar{w}_{k}^{i}}{\sum_{i}^{N_{p}} \bar{w}_{k}^{i}}
$$

and $\delta(\cdot)$ denotes the Dirac delta function.

In the Bayesian recursion, a common proposal density can be chosen as $p\left(\mathbf{x}_{1: k}^{t} \mid \mathbf{z}_{1: k}\right)=p\left(\mathbf{x}_{k}^{t} \mid \mathbf{x}_{k-1}^{t}\right) p\left(\mathbf{x}_{1: k}^{t} \mid \mathbf{z}_{1: k-1}\right)$. This means that given the samples $\left\{\mathbf{x}_{1: k-1}^{i}, w_{k-1}^{i}\right\}_{i=1}^{N_{p}}$ at $k-1$, new samples can be drawn as $\mathbf{x}_{k}^{i} \sim p\left(\mathbf{x}_{k} \mid \mathbf{x}_{k-1}^{t, i}\right)$ and attached to the existing sample set. Substituting Eq. (5) into Eq. (7) gives the corresponding update on weights $w_{k}^{i} \propto w_{k-1}^{i} p\left(\mathbf{z}_{k} \mid \mathbf{x}_{k}^{t, i}\right)$.

In addition to the above general particle filtering framework, in some target tracking scenarios, extra knowledge about the system state may be incorporated to improve the estimation accuracy. Specifically in this paper, information about the road constraint is considered, which usually limits the vehicle's movement within a specified space defined by $\mathbf{g}\left(\mathbf{x}_{k}^{t}\right) \leq 0$ where $\mathbf{g}(\cdot)$ is a possibly nonlinear function. To incorporate this inequality constraint, the sampling step is augmented with the acceptance-rejection process [9], which accepts the sample only if it satisfies the constraint. The corresponding constrained particle filter algorithm is summarised in Algorithm 1.

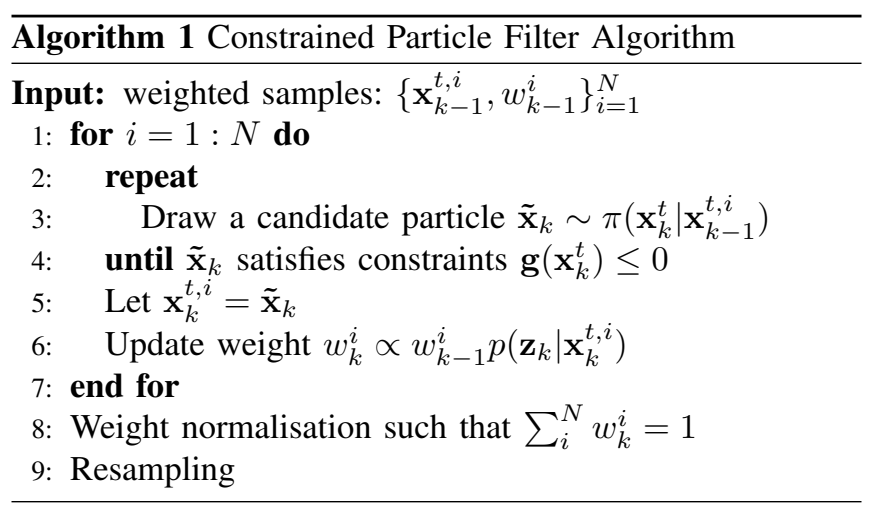

\section{B. Sensor fusion}

In case that there are multiple UAVs involved in tracking of the same target, sensor fusion techniques can be utilised to improve the estimation accuracy. In this study, simple measurement fusion is adopted which utilises an augmented measurement vector with other UAV's sensor measurements obtained via communication (i.e. $\mathbf{z}_{k}=\left[\begin{array}{llll}\mathbf{z}_{k}^{1} & \mathbf{z}_{k}^{2} & \cdots & \mathbf{z}_{k}^{m}\end{array}\right]^{T}$ where $m$ is the number of UAVs involved) for weight update in Algorithm 1. Note that other sensor fusion techniques can also be utilised [10].

\section{Prediction of an out-of-surveillance target}

To ensure that the UAV tracks the target persistently, it is required to predict the states of the target with a certain error bound even in case of out of surveillance (in turn, target loss due to sensing failure or line-of-sight block by obstacles). The CPF can inherently provide the possible position of the lost target by propagating sampled particles with the target dynamics within the road in the prediction (sampling) stage in Algorithm 1. Figure 1 shows the comparison of out-ofsurveillance target prediction examples with different estimation methods. In this figure, inner circles are determined by the position error covariance (a green ellipsoid) from the extended Kalman filter (EKF) or the generated particles with the estimated target position from the PF and CPF. Outer circles represent the standoff orbit to be followed by the 


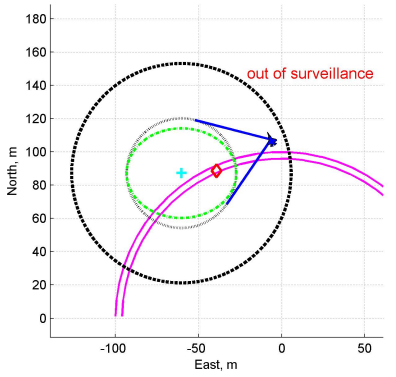

(a) Extended Kalman filter

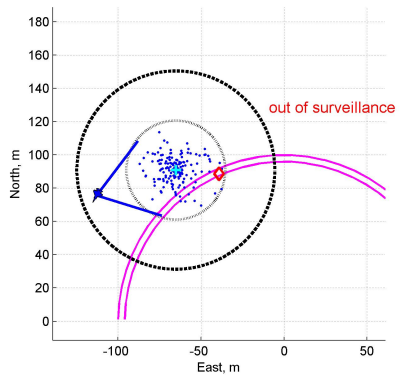

(b) Particle filter

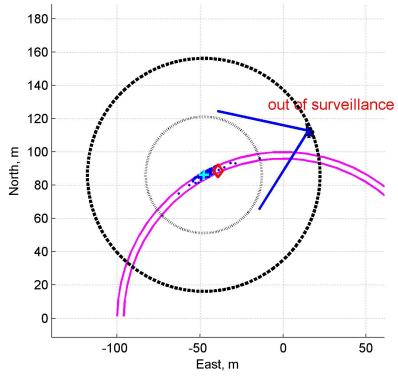

(c) Constrained Particle filter

Fig. 1. Comparison of out-of-surveillance target position prediction using different estimation methods (blue small dots: particles from the particle filter, cyan plus symbol: estimated target position, and red diamond: true target position).

UAV ensuring that the inner circle is within the sensor FOV (blue lines). Apparently, we could get a better picture for the possible position of the lost target (i.e. the intersection of the inner circle and the road) using the CPF. Even though the error covariance matrix of the EKF could be projected into the road-constrained subspace, this projection is not straightforward given that the road is nonlinear. Note that the number of particles used for the CPF is directly related to the size of the error bound, which should be carefully determined according to the fidelity of target dynamics.

\section{Coordinated Standoff TARget TRACKInG}

Once the possible existence area of the in- or out-ofsurveillance target is determined from the CPF, coordinated standoff tracking guidance is performed by UAVs. The objective of the guidance is to keep all particles within the fieldof-view (FOV) of the UAV sensor by commanding UAVs to maintain corresponding distances (standoff distance) from the estimated target position. To this end, this study proposes to use the Lyapunov vector field guidance (LVFG) and the nonlinear model predictive control (NMPC) concept.

\section{A. UAV Dynamics}

Assuming each UAV has a low-level flight controller (autopilot), this study aims to design guidance inputs to this low-level controller for standoff target tracking. Consider a two-dimensional UAV kinematic model [7] as:

$$
\left(\begin{array}{c}
\dot{x} \\
\dot{y} \\
\dot{\psi} \\
\dot{v} \\
\dot{\omega}
\end{array}\right)=f(\mathbf{x}, \mathbf{u})=\left(\begin{array}{c}
v \cos \psi \\
v \sin \psi \\
\omega \\
-\frac{1}{\tau_{v}} v+\frac{1}{\tau_{v}} u_{v} \\
-\frac{1}{\tau_{\omega}} \omega+\frac{1}{\tau_{\omega}} u_{\omega}
\end{array}\right)
$$

where $\mathbf{x}=(x, y, \psi, v, \omega)^{T}$ are the inertial position, heading, speed and yaw rate of the UAV, respectively. $\tau_{v}$ and $\tau_{\omega}$ are time constants for considering actuator delay. $\mathbf{u}=\left(u_{v}, u_{\omega}\right)^{T}$ are the commanded speed and turning rate constrained by the following dynamic limits of fixed-wing UAV: $\left|u_{v}-v_{0}\right| \leq$ $\Delta v_{\max }$ and $\left|u_{\omega}\right| \leq \omega_{\max }$ where $v_{0}$ is a nominal speed of UAV. The continuous UAV model in Eq. (8) can be discretised by Euler integration into:

$$
\mathbf{x}_{k+1}=f_{d}\left(\mathbf{x}_{k}, \mathbf{u}_{k}\right)=\mathbf{x}_{k}+T_{s} f\left(\mathbf{x}_{k}, \mathbf{u}_{k}\right)
$$

where $\mathbf{x}_{k}=\left(x_{k}, y_{k}, \psi_{k}, v_{k}, \omega_{k}\right)^{T}, \mathbf{u}_{k}=\left(u_{v k}, u_{\omega k}\right)^{T}$, and $T_{s}$ is a sampling time. Note that if the frequencies of the guidance law and autopilot are not too close, it is common to initially design and verify the guidance law and control algorithm separately. Therefore, like in many literatures considering similar guidance problems [4], [7], [11], above simple kinematics is used for the UAV model.

\section{B. LVFG with variable standoff distance}

The LVFG uses the following vector field function to compute the desired velocity to guide the UAV to orbit around the ground target [4]: $V_{l}(x, y)=\left(r^{2}-r_{d}^{2}\right)^{2}$. Then, the total time derivative of $V_{l}$ is given by $\dot{V}_{l}=\nabla V \cdot[\dot{x}, \dot{y}]^{T}$, and the following desired velocity $\left[\dot{x}_{d}, \dot{y}_{d}\right]^{T}$ can make $\dot{V}_{l}$ nonpositive, which provides stable convergence to the circling limit cycle around the target as shown in Fig. 2 (in this subsection, the subscript $k$ will be omitted for simple notation):

$$
\left[\begin{array}{c}
\dot{x}_{d} \\
\dot{y}_{d}
\end{array}\right]=\frac{-v_{d}}{r\left(r^{2}+r_{d}^{2}\right)}\left[\begin{array}{c}
\delta x\left(r^{2}-r_{d}^{2}\right)+\delta y\left(2 r r_{d}\right) \\
\delta y\left(r^{2}-r_{d}^{2}\right)-\delta x\left(2 r r_{d}\right)
\end{array}\right]
$$

where $\delta x=x-x^{t}, \delta y=y-y^{t}$, and $r=\sqrt{\delta x^{2}+\delta y^{2}}$ is the distance between the UAV and the target. Herein $\left(x^{t}, y^{t}\right)$ is the target position estimated from the tracking filter, and $v_{d}$ is a desired UAV speed. Loitering direction in this study is clockwise as shown in Fig. 2, and it can be changed by using different sign in Eq. (10). $r_{d}$ is a desired standoff distance from the UAV to the target which can be computed considering the FOV $\alpha_{f}$ of the UAV as:

$$
r_{d}=\frac{d_{\max }}{\sin \left(\frac{\alpha_{f}-\varepsilon_{m}}{2}\right)}
$$

where $d_{\max }$ is the distance between the estimated target position and the particle furthest from the target as shown in Fig. 2, and $\varepsilon_{m}$ is an angle margin for the FOV of the UAV. Note that $r_{d}$ should be bounded by the physical (turning) constraints the UAV, thus $r_{d} \geq r_{d, \text { min }}$. Convergence analysis of vector field to the standoff orbit can be found in [12].

By commanding the UAV to maintain the standoff distance, the UAV can keep all the particles within its FOV as shown in Fig. 3. The guidance command $u_{\omega}$ for UAV turn 


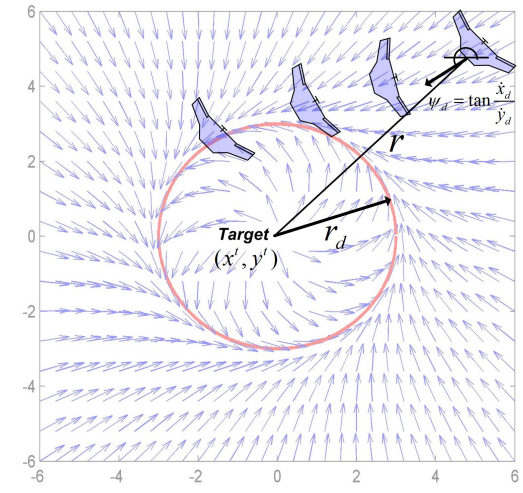

Fig. 2. Illustration of vector field and standoff orbit tracking.

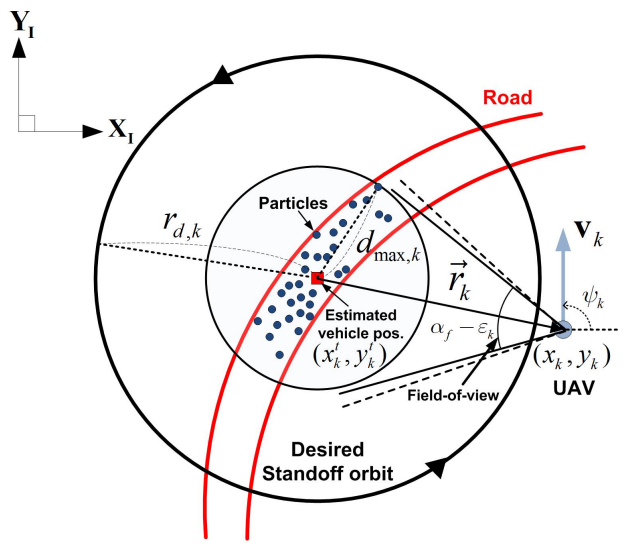

Fig. 3. Geometric relation between UAV, particles from the CPF, and estimated target position

rate is computed by proportional feedback and feedforward control terms as:

$$
u_{\omega}=-k_{\omega}\left(\psi-\psi_{d}\right)+\dot{\psi}_{d}
$$

where the desired heading $\psi_{d}$ can be determined using the desired velocity in Eq. (10) as: $\psi_{d}=\tan ^{-1}\left(\dot{y}_{d} / \dot{x}_{d}\right)$ and differentiating $\psi_{d}$ gives:

$$
\dot{\psi}_{d}=4 v_{d} \frac{r_{d} r^{2}}{\left(r^{2}+r_{d}^{2}\right)^{2}}-\frac{2 r \dot{r}_{d}}{r^{2}+r_{d}^{2}} .
$$

Note that for the guidance command to be feasible (i.e. within $\omega_{\max }$ ), the control gain $k_{\omega}$ and $r_{d, \min }$ need to be carefully determined.

In case that a pair of UAVs are used to track the same target while flying on the same orbit, the angular separation between UAVs needs to be maintained in order to obtain more accurate target information as well as to avoid collision. Among various approaches [4], [13], this study uses speed control of each UAV:

$$
u_{v}= \pm k_{v}\left(\gamma-\theta_{d}\right) r_{d}+v_{d}
$$

where $k_{v}$ is a control gain, $\gamma$ is the angular phase separation between UAVs, and $\theta_{d}$ is a desired phase difference between the UAVs. Note that the guidance algorithm is separated to standoff orbit tracking by the heading rate $u_{\omega}$ and phase angle maintenance by the speed control $u_{v}$, which is termed as a decoupled structure.

\section{NMPCST with variable standoff distance}

This subsection proposes to use a nonlinear model predictive control (NMPC) framework [7] for coordinated standoff tracking. Unlike a decoupled control structure as in the LVFG, the NMPC is a coupled approach where the heading rate and speed control contribute to both standoff orbit tracking and phase angle control, which provides a suboptimal performance in terms of tracking accuracy as well as control efforts. Since the NMPC utilises future states of the target as well as UAVs within the receding horizon steps, the prediction of the target movement plays a more important role on the tracking guidance performance.

The model predictive coordinated standoff tracking (NMPCST) decides a control input sequence for $N$ sampling times that minimises the following performance index for maintaining a distance between a UAV and a ground target as well as a relative phase angle between UAVs.

$$
\begin{aligned}
J= & \phi\left(\tilde{r}_{N}, \tilde{d}_{N}\right)+\sum_{k=0}^{N-1} L\left(\tilde{r}_{k}, \tilde{d}_{k}, \mathbf{u}_{\mathbf{k}}\right) \\
\phi\left(\tilde{r}_{N}, \tilde{d}_{N}\right)= & \frac{1}{2}\left(p_{r} \tilde{r}_{N}^{2}+p_{d} \tilde{d}_{N}^{2}\right) \\
L\left(\tilde{r}_{k}, \tilde{d}_{k}, \mathbf{u}_{\mathbf{k}}\right)= & \frac{1}{2}\left\{q_{r} \tilde{r}_{k}+q_{d} \tilde{d}_{k}^{2}+r_{v}\left(\frac{u_{v k}-v_{d}}{v_{\max }}\right)^{2}+\right. \\
& \left.r_{\omega}\left(\frac{u_{\omega k}-\frac{v_{d}}{r_{d, m i n}}}{\omega_{\max }}\right)^{2}\right\}
\end{aligned}
$$

where $\tilde{r}_{k}=\left(r_{d, k}^{2}-\left|\boldsymbol{r}_{k}\right|^{2}\right) / r_{d, k}^{2}$ and $\tilde{d}_{k}=\left(\boldsymbol{r}_{k}^{T} \boldsymbol{r}_{k}^{p}+\right.$ $\left.\left|\boldsymbol{r}_{k}\right|\left|\boldsymbol{r}_{k}^{p}\right|\right) / r_{d, k}^{2}$ with $\boldsymbol{r}_{k}$ and $\boldsymbol{r}_{k}^{p}$ represent the relative vectors from the target position to the positions of the current UAV and its pair UAV, respectively. On computing $\boldsymbol{r}_{k}$ up to $N$ steps, the future position of the target and the UAV is predicted by their dynamic models, and for the target position outside the road, a simple interior point method [14] is used to bring it back into the constrained region. $r_{d, k}$ is a desired standoff distance from the UAVs to the target position as defined in Eq. (11). $p_{r}, p_{d}, q_{r}, q_{d}, r_{v}$, and $r_{\omega}$ are constant weighting scalars. $\tilde{d}_{k}$ is derived from the inner product of $\boldsymbol{r}_{k}$ and $\boldsymbol{r}_{k}^{p}$ as $<\boldsymbol{r}_{k}, \boldsymbol{r}_{k}^{p}>=\boldsymbol{r}_{k}^{T} \boldsymbol{r}_{k}^{p}=\left|\boldsymbol{r}_{k}\right|\left|\boldsymbol{r}_{k}^{p}\right| \cos \triangle \theta_{k}$ where $\Delta \theta_{k}=\left|\theta_{k}^{p}-\theta_{k}\right|$ with the phase angles of UAV positions with respect to the current target location. If the phase difference $\Delta \theta_{k}$ is ideally maintained as $\pi$ radian, the above equation is rearranged as $\boldsymbol{r}_{k}^{T} \boldsymbol{r}_{k}^{p}+\left|\boldsymbol{r}_{k}\right|\left|\boldsymbol{r}_{k}^{p}\right|=0$. Therefore, $\tilde{d}_{k}$ can be used to ensure phase angle keeping between UAVs. By incorporating the UAV dynamics in Eq. (9) and admissible control input ranges as equality and inequality constraints, an augmented performance index can be derived as:

$$
\begin{aligned}
& J_{a}=\phi\left(\tilde{r}_{N}, \tilde{d}_{N}\right) \\
& +\sum_{k=0}^{N-1}\left[L\left(\tilde{r}_{k}, \tilde{d}_{k}, \mathbf{u}_{k}\right)+\lambda_{k+1}^{T}\left\{f_{d}\left(\mathbf{x}_{k}, \mathbf{u}_{k}\right)-\mathbf{x}_{k+1}\right\}\right. \\
& \left.+\frac{1}{2} \mu_{v} l_{v k} S_{v}^{2}\left(\mathbf{u}_{k}\right)+\frac{1}{2} \mu_{\omega} l_{\omega k} S_{\omega}^{2}\left(\mathbf{u}_{k}\right)+\frac{1}{2} \mu_{c} l_{c k} S_{c}^{2}\left(\mathbf{x}_{k}\right)\right]
\end{aligned}
$$


where $S_{v}\left(\mathbf{u}_{k}\right)=\frac{\left|u_{1 k}-v_{d}\right|-v_{\max }}{v_{\max }} \leq 0, S_{\omega}\left(\mathbf{u}_{k}\right)=$ $\frac{\left|u_{2 k}\right|-\omega_{\max }}{\omega_{\max }} \leq 0, S_{c}\left(\mathbf{x}_{k}\right)=\frac{r_{c}-\left|C\left(\mathbf{x}_{k}-\mathbf{x}_{k}^{p}\right)\right|}{r_{c}} \leq 0, r_{c}$ is a safe distance between the UAVs to prevent collision, $\lambda_{k}$ is a Lagrange multiplier, and $\mu_{v}, \mu_{\omega}$, and $\mu_{c}$ are penalty function parameters. $l_{v k}, l_{\omega k}$, and $l_{c k}$ are defined to avoid unnecessary computation for satisfying inequality constraints:

$$
l_{* k}=\left\{\begin{array}{cc}
0, & S_{*} \leq 0 \\
1, & S_{*}>0 .
\end{array} .\right.
$$

Then, gradient-descent based online optimisation can be performed to find an optimal solution for above performance index. Each UAV runs the optimization routine in flight in a decentralized way at each sampling while getting necessary information of others via communications.

\section{NumERicAl Simulations}

This section carries out numerical simulations using the proposed standoff tracking algorithms with the constrained PF for a moving ground target. The ground target is assumed to be moving on the road defined by two arcs with radius of $r_{1}=96 \mathrm{~m}$ and $r_{2}=100 \mathrm{~m}$ with the following dynamics:

$$
\mathbf{x}_{k}^{t}=\left[\begin{array}{cccc}
1 & 0 & T_{s} & 0 \\
0 & 1 & 0 & T_{s} \\
0 & 0 & 1 & 0 \\
0 & 0 & 0 & 1
\end{array}\right] \mathbf{x}_{k-1}^{t}+\left[\begin{array}{cc}
0.5 T_{s}^{2} & 0 \\
0 & 0.5 T_{s}^{2} \\
0 & 0 \\
0 & 0
\end{array}\right] \mathbf{w}_{k}
$$

where $\mathbf{x}_{k}^{t}=\left[x_{k}^{t}, y_{k}^{t}, \dot{x}_{k}^{t}, \dot{y}_{k}^{t}\right]^{T}$ and $\mathbf{w}_{k}$ is a 2-D Gaussian process noise with zero mean and identity covariance matrix. Given the road boundaries above, the road constraint can be expressed as: $r_{1} \leq \sqrt{\left(x_{k}^{t}\right)^{2}+\left(y_{k}^{t}\right)^{2}} \leq r_{2}$. Besides, UAVs are equipped with an onboard ground moving target indicator sensor to localise the position of target where the measurement $\mathbf{z}_{k}=(r, \theta)^{T}$ can be defined as:

$$
\mathbf{z}_{k}=\left(\begin{array}{c}
\sqrt{\left(x_{k}^{t}-x_{k}\right)^{2}+\left(y_{k}^{t}-y_{k}\right)^{2}} \\
\tan ^{-1} \frac{y_{k}^{t}-y_{k}}{x_{k}^{t}-x_{k}}
\end{array}\right)+\mathbf{v}_{k}
$$

where $\mathbf{v}_{k}$ is a measurement noise vector, and its noise covariance matrix $R_{k}=\operatorname{diag}\{100,0.01\}$. The sample vehicle trajectory for 70 seconds is shown in Fig. 4, and the target is assumed to be out of surveillance for $20 \sim 30$ and $45 \sim 55$ seconds, unless otherwise stated. For performance analysis, Monte Carlo simulations with a hundred independent runs were performed. The other setting of simulation parameters needed for guidance algorithms can be found in Table I.

The effect of the number of particles used for the CPF on the standoff distance and the estimation error is investigated considering the out-of-surveillance period only in the scenario, as shown in Fig. 5. As the more particles are used, the wider they are spatially dispersed, the standoff distance increases accordingly (by $d_{\max }$ in (11) ) which also increases the chance of putting the target within the FOV. However, since this could increase the computation time and make the standoff orbit expand too fast to be followed by the UAV, this study uses $N_{p}=200$.

The target tracking performance of the LVFG is investigated with different estimation methods as shown in Table II.

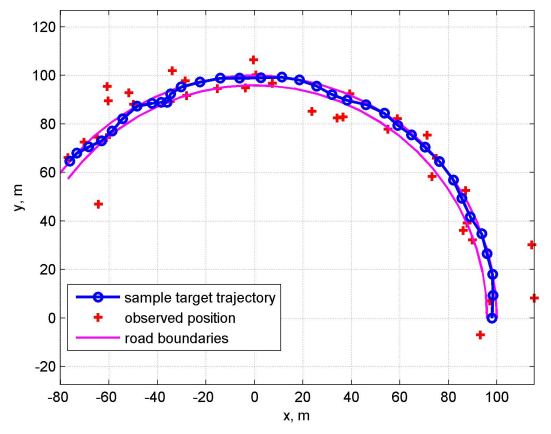

Fig. 4. Ground vehicle tracking scenario and a sample trajectory.

TABLE I

SIMULATION PARAMETERS

\begin{tabular}{llc} 
Parameter & Value & Unit \\
\hline$T_{s}$ & 0.5 & $\mathrm{sec}$ \\
$N, N_{p}$ & 6,200 & $\mathrm{~N} / \mathrm{A}$ \\
$\theta_{d}$ & $\pi$ & $\mathrm{rad}$ \\
$v_{d}$ & 15 & $\mathrm{~m} / \mathrm{s}$ \\
$\left(r_{d, \min }, r_{d, \max }\right)$ & $(30,100)$ & $\mathrm{m}$ \\
$\Delta V_{\max }$ & 7 & $\mathrm{~m} / \mathrm{s}$ \\
$\omega_{\max }$ & 0.7 & $\mathrm{rad} / \mathrm{s}$ \\
$\tau_{v}, \tau_{\omega}$ & $1 / 3$ & $\mathrm{sec}$ \\
$\left(\alpha_{f}, \epsilon_{m}\right)$ & $(80,20)$ & $\mathrm{deg}$ \\
$\left(p_{r}, p_{d}, q_{r}, q_{d}, r_{v}, r_{\omega}\right)$ & $\left(500,100, p_{r} / N, p_{d} / N, 100,50\right)$ & $\mathrm{N} / \mathrm{A}$ \\
$\left(k_{\omega}, k_{v}, k_{l}\right)$ & $(0.5,1.2,0.8)$ & $\mathrm{N} / \mathrm{A}$ \\
$\mu_{v}, \mu_{\omega}$ & $1 e 3$ & N/A
\end{tabular}

The CPF improves the estimation accuracy significantly with the help of road information compared to the PF, and consequently reduces tracking guidance error and target loss. Here, target loss represents the number of incidents where target goes outside the FOV out of 100 simulation runs. The use of multiple UAVs with sensor fusion $\left(\mathrm{CFP}_{\text {multi }}\right)$ in the particle filtering further improves the tracking performance.

Table III compares the performance of the LVFG and the NMPCST using two UAVs. In the table, 'In' and 'In/Out' represent the simulation scenarios where the target is always in-surveillance and in and out of surveillance, respectively. The control efforts on $u_{v}$ and $u_{\omega}$ are computed by integrating the time histories of $\left|u_{v}-v_{d}\right|$ and $\left|u_{\omega}\right|$, respectively. For the 'In' scenario, since target estimation

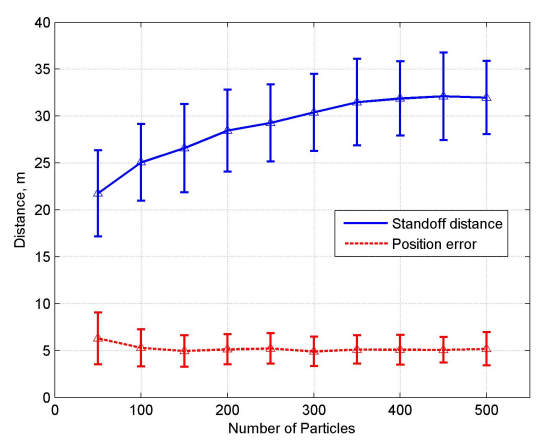

Fig. 5. Standoff distance prediction with respect to the number of particles 
TABLE IV

COMPARISON BETWEEN LVFG AND NMPCST

\begin{tabular}{lll}
\hline & LVFG & NMPCST \\
\hline \hline Prediction & One-step look ahead & Receding horizon steps \\
Control methodology & Decoupled for distance and phase angle & $\begin{array}{l}\text { Coupled } \\
\text { Sub-optimal (better suited for nonlinear }\end{array}$ \\
Tracking performance & Limited & \& non-Gaussian road constraints) \\
Loitering direction & Fixed & Variable \\
Convergence & Guaranteed & Subject to optimisation conditions \\
Parameter tuning & Simple & Complex \\
Collision \& Obs. avoidance & - & Simply adding more constraints in $J_{a}(17)$ \\
Computation & Light & Heavy (albeit approximation is possible) \\
\hline
\end{tabular}

TABLE II

PERFORMANCE COMPARISON BETWEEN DIFFERENT ESTIMATION METHODS USING THE LVFG

\begin{tabular}{lccc}
\hline \multirow{2}{*}{ Mean error } & \multicolumn{2}{c}{ Single UAV } & Multiple UAVs \\
& PF & CPF & CPF $_{\text {multi }}$ \\
\hline \hline Position $(\mathrm{m})$ & $7.29(12.90)$ & $3.94(6.04)$ & $2.38(4.25)$ \\
Velocity $(\mathrm{m} / \mathrm{s})$ & $1.79(2.39)$ & $1.29(1.64)$ & $1.05(1.43)$ \\
Standoff distance $(\mathrm{m})$ & 13.34 & 8.84 & 6.78 \\
Phase keeping $(\mathrm{deg})$ & - & - & 12.04 \\
Target loss & 20 & 13 & 1 \\
\hline \multicolumn{2}{l}{$(\cdot)$ represents the result for the period of out-of-surveillance only }
\end{tabular}

TABLE III

TRACKING PERFORMANCE WITH DIFFERENT GUIDANCE METHODS

\begin{tabular}{lcccc}
\hline \multirow{2}{*}{ Mean error } & \multicolumn{2}{c}{ LVFG } & \multicolumn{2}{c}{ NMPCST } \\
& In & In/Out & In & In/Out \\
\hline \hline Standoff distance $(\mathrm{m})$ & 3.60 & 6.78 & 3.29 & 6.97 \\
Phase keeping $(\mathrm{deg})$ & 11.20 & 12.04 & 9.25 & 13.94 \\
$u_{v}$ control effort $(\mathrm{m} / \mathrm{s})$ & 69.80 & 79.98 & 71.18 & 117.08 \\
$u_{\omega}$ control effort $(\mathrm{rad} / \mathrm{s})$ & 31.10 & 29.34 & 30.76 & 29.87 \\
\hline
\end{tabular}

is quite accurate and as a result, the standoff distance is almost constant (i.e. $r_{d}=r_{d, \text { min }}$ ), the NMPCST shows better performance thanks to the optimisation with future prediction over multiple time steps. Note that the LVFG uses basically a one-step look ahead approach. However, for the 'In/Out' case, whenever the target goes out of surveillance (20 30 and 45 55 seconds as mentioned) and then comes back to in surveillance, the standoff distance and the estimated target position are subject to sudden changes, which makes the performance of the prediction-based NMPC worse than that of the LVFG. Also, note that the loitering direction is not specified in the NMPC formulation (as opposed the LVFG which uses the fixed direction), so it could be changed even in the middle of the same target tracking mission; this leads to more tracking error and excessive control efforts as shown in the Table III and movie clips which can be downloaded at https://dl.dropboxusercontent.com/u/17047357/CST.zip. Lastly, Table IV summarises characteristics of the LVFG and the NPMCST approach.

\section{CONClusions And Future WORK}

This paper has presented the standoff target tracking guidance framework for multiple UAVs using the particle filtering approach aided by roadmap information. Numerical simulation shows the feasibility and benefit of the proposed approach and also compared two guidance algorithms: the LVGF and the NMPCST. It was shown that both algorithms have their own merit depending on the situation. Extension of the constrained particle filter to the multiple model filter considering road junctions and different vehicle models will be followed as future work along with allocating multiple UAVs intelligently to the corresponding road segments.

\section{REFERENCES}

[1] Z. Tang and U. Ozguner. PF-HMap: A target track maintenance approach for mobile sensor platforms with intermittent and regional measurements. In IEEE Conference on Decision and Control, San Diego, USA, 2006.

[2] J.G. Herrero, J.A.B. Portas, and J.R.C. Corredera. Use of map information for tracking targets on airport surface. IEEE Transactions on Aerospace and Electronic Systems, 39(2):675-693, 2003.

[3] M.S. Arulampalam, N. Gordon, M. Orton, and B. Ristic. A variable structure multiple model particle filter for GMTI tracking. International Conference on Information Fusion, 2002.

[4] E.W. Frew, D.A. Lawrence, and S. Morris. Coordinated standoff tracking of moving targets using Lyapunov guidance vector fields. Journal of Guidance, Control, and Dynamics, 31(2), 2008.

[5] H. Oh, S. Kim, H.S. Shin, B.A. White, A. Tsourdos, and C.A. Rabbath. Rendezvous and standoff target tracking guidance using differential geometry. Journal of Intelligent and Robotic Systems, 69:389-405, 2013.

[6] R. Sepulchre, D.A. Paley, and N.E. Leonard. Stabilization of planar collective motion: All-to-all communication. IEEE Transactions on Automatic Control, 52(5):811-824, 2007.

[7] S. Kim, H. Oh, and A. Tsourdos. Nonlinear model predictive coordinated standoff tracking of moving ground vehicle. Journal of Guidance, Control and Dynamics, 36(2):557-566, 2013.

[8] M.S. Arulampalam, S. Maskell, N. Gordon, and T. Clapp. A tutorial on particle filter for online nonlinear/non-Gaussian bayesian tracking. IEEE Transations on Signal Processing, 50:172-188, 2002.

[9] L. Lang, W.S. Chen, B.R. Bakshi, P.K. Goel, and S. Ungarala. Bayesian estimation via sequential Monte Carlo sampling constrained dynamic systems. Automatica, 43:1615-1622, 2007.

[10] R.R. Brooks and S.S. Iyengar. Multi-sensor Fusion: Fundamentals and Applications with Software. Prentice Hall PTR, Upper Saddle River, NJ, 1997.

[11] D. Kingston and R. Beard. UAV spaly state configuration for moving targets in wind. Lecture Notes in Control and Information, 369:109$128,2007$.

[12] H. Oh, S. Kim, H. Shin, A. Tsourdos, and B. White. Coordinated standoff tracking of moving target groups using multiple UAVs. IEEE Transactions on Aerospace and Electronic Systems, 2015. accepted.

[13] H. Oh, S. Kim, A. Tsourdos, and B.A. White. Decentralised standoff tracking of moving targets using adaptive sliding mode control for UAVs. Journal of Intelligent and Robotic System, 76:169-183, 2014.

[14] S.P. Boyd and L. Vandenberghe. Convex Optimization. Cambridge Univer, 2004. 